\title{
LXIX. On short-hand writing
}

\section{H. Upington Esq.}

To cite this article: $\mathrm{H}$. Upington Esq. (1823) LXIX. On short-hand writing, Philosophical Magazine Series 1, 61:301, 325-330, DOI: 10.1080/14786442308644332

To link to this article: http://dx.doi.org/10.1080/14786442308644332

曲 Published online: 29 Jul 2009.

Submit your article to this journal 중

III Article views: 3

Q View related articles $\sqsubset$ 
It would be desirable to know the comparative deficiency of rain at every 8 or 10 feet above the level of the ground, up to 100 feet, which perhaps is as high as any rain-gauge is placed upon buildings, in order to be clear of all obstructions. But when there are so many collateral circumstances to be taken into the account, it seems very difficult to determine upon a constant allowance for any proposed height, except by careful experiment, or the practical results of every day's rain through the whole year. So long as this remains one of the grand arcana in meteorology, and the height of the rain-gauges is not particularly mentioned, it is impossible that we can know the real product of rain at the ground in the different parts of the country where rain-gauges are kept: indeed, I much fear, from this necessary precaution not having been verv particularly attended to, that many of the laborious results of rain caught and published are far from being what they ought to be; namely, the correct annual quantities that have fallen at or near the surface of the earth.

It is probable that these remarks, if they should be deemed worth inserting in your valuable Magazine, will be contested by some of your able correspondents: if so, I trust they will first take a little trouble, as I have done, to draw their conclusions from practical results.

Yours, \&c.

Glosterian.

LXIX. On Short-hand Writing. By H. UpIngton, Esq.

[Continued from vol. lix. p. 28.]

Blair's Hill Cork, Feb. 13.

I SHOULD hope that by this time I have thoroughly satisfied my reader, that nothing within my power has been left untried to convey to him a comprehensive idea of my design. I shall now proceed in the same manner I have hitherto done, and lay before him, previously to the production of my alphabet, the method which I pursued in its formation.

I first directed my attention towards the "scale of characters," and found, that of the first series or right lines there were but three remaining; having, for the sake of muscular execution and the promotion of lineality, relinquished both the diagonal descending ones $/$ and 1 , and substituted in their stead * the looped characters $\sigma$ equal to $\sigma$, and $\sigma=e$. The first [pair] being somewhat less complex than the second, and contributing much, by its ascending direction, to counter-

* The loss occasioned by this substitution has been already calculated. 
act the descending tendency of our short-hand words, I allotted to the letter $\mathrm{L}$, which is the sixth letter on the "scale of occurrence." And to the letter K, which is only the eleventh upon the scale, I allotted the less simple though at the same time the more lineal [or level] one.

The question then was, How should the three remaining right lines be appropriated? Apparently to the three first letters N T S. But here all fixed rules of brevity gave way in some trifling degree to convenience. In our language there are frequently so many repetitions of $\mathrm{N}$ and $\mathrm{S}$ to be found in the same word, that lineal [call them horizontal] characters are desirable for both ; and therefore to $\mathrm{N}$ I assigned the most eligible of the simple curves $\smile$; and to $S^{*}$ the horizontal line -

$T$ was provided for by the perpendicular $\mid$; and $R$ (the fourth letter of the scale) obtained the ascendingt diagonal $\%$.

Three additional curves were now to be disposed of; one of which, viz. ), I bestowed upon the succeeding letter $D$. L was already formed. Th and $F$ were passed over for reasons I shall immediately adduce. $M$ obtained another of those curves, viz. the lineal [horizontal] one $\frown$; while the remaining one ( being in many cases very inconvenient for junction with preceding characters, was assigned not to $\mathrm{P}$ [K was already disposed of ], but to the following letter $B$, which occurs somewhat oftener than $\mathrm{P}$ as an incipient, but far less frequently than $\mathrm{P}$ as a subsequent.

I now return to Th and $\mathrm{F}$. Th (which I shall almost banish as an intermediate, and express by $T$ aspirated when necessary,) is the second letter on our scale as an incipient. To it then I assigned the most suitable of the third class or hooked characters, viz. $]$, or at option $\int$. These characters, though swift, and convenient as incipients, are in many cases extremely difficult of precise execution as intermediates.

$\mathbf{F}$, although a little superior to $M$ on the scale of occurrence, obtained the remaining $\ddagger$ hooked characters $-=-$; which characters, notwithstanding the peculiar combinations

* As the conjunction " and" shall hereafter be represented by an arbitrary; and as the letter $\mathrm{Sh}$ is to be expressed by aspirated $\mathrm{S}$; the difference between $N$, otherwise $=1000$, and $S=762$, will be so diminished, that, in order to avoid some awkward turns, I conferred the horizontal line upon the letter $S$ in preference to $N$.

+ Although $\mathrm{N}$ has been reduced in brevity by the assignment of a curve in place of a right line; yet $R$, to which this curve should otherwise have fallen, has been consequently increased. Therefore the actual loss sustainable is infinitely less than the superficial observer may suppose.

‡ I say " remaining, " in consequence of my having expunged as general letters (for the prevention of crabbed turns and difficult angles) the hooked char acters descending to the right. 
of our language, might indeed with almost equal muscular advantage, and perhaps some fractional advantage as to brevity, have been assigned to $M$ : but when I considered that subsequent $\mathrm{V}$ is sometimes to be expressed by $\mathrm{F}, \mathrm{I}$ thought it desirable that the double character should be given to $\mathrm{F}$ in preference to $M$, in order that $V$ should, on such occasions, be rendered more definite. I shall treat of this distinction in the sequel.

The next characters of which I have to speak, are the two simple looped* ones $\mathrm{P}=9$ and $\sigma=a$, taking care, as I already stated, that the incipient disadvantage of these shall be obviated by prepositives. These characters then have been regularly applied, in the order of occurrence, to those letters which most require them as subsequents: the former to the letter $P$; to $G$ the latter.

$\mathrm{V}$, which I passed unnoticed in its proper place, does not obtain the only remaining and comparatively tedious character $e=9$; but is represented when incipient or alone, by the two simple lines $\mathrm{V}$. When a subsequent, it is similarly written in convenient cases; and in other cases it is expressed (often definitely) by the letter $\mathrm{F}$.

W. This letter, which rates considerably above $\mathrm{V}$ as an incipient, [when a subsequent which very rarèly occurs, it is considered as a voroel, ] I designated by one of the quickest and most convenient of the rejected hooked characters; viz. $\checkmark$.

H. I call this letter an aspirate, and provide for it among the vowels, thus '.

Wh, incipient, is expressed by the only eligible character remaining among the rejected hooks, viz. 7 ; the line, to avoid angles, being in all difficult cases, somewhat inflected, thus V. Wh, when a subsequent, which very rarely happens, is omitted like W.

$\bar{Y}$, which scarcely ever occurs as an incipient $\uparrow$ [when a subsequent, it is ranked among the vowels], I have represented by the comparatively tedious mixed character $\checkmark$ which is well calculated for junction with succeeding ones.

Thr as an incipient is expressed by $T h$ and $R, V$ : when a subsequent by $\mathrm{T}$ and $\mathrm{R}$, thus $\mathrm{V}$. The aspirate, if thought necessary, may subsequently be introduced as thus $\dot{V}$.

* The ascending diagonal one was already conferred on $\mathrm{L}$; and the descending one towards the right has been expunged as a general character for reasons too often mentioned.

+ "You," "your," which in our language cause the frequency of the letter $y$ as an incipient, shall be otherwise disposed of. Does not this letter alone expose the fallacy of our system-mongers who style their plans of short-hand "universal?" Every language has its own peculiar character. 
Ch cannot be dispensed with either as an incipient or subsequent. As an incipient it is very rare. The only remaining and least desirable of all the looped characters, viz. $e=9$, is therefore appropriated to this double letter.

Sh. $\mathbf{S}$ serves for the basis of this double letter. The aspirate may be omitted or used at option; thus - when incipient or intermediate; thus - when final or alone.

$\mathrm{X}$ is represented by two detached horizontal lines; as thus -; the upper line being joined to the succeeding character, as "extent" 7 i. e. $x$ tnt.

$Q$ is expressed, when incipient or alone, by the two right lines $\%$ The horizontal one being last formed is joined to the following character like the upper line of the letter $\mathrm{X}$. When $Q$ is a subsequent, $\mathrm{K}$ is used in the place of it; and should obscurity be apprehended, the letter $W$ is added, as thus with the word "request" (written rkrost) פัح.

J. Soft $\mathrm{G}$ being in all cases sounded like this letter, $\mathrm{G}$ is always substituted for it.

$\mathrm{Z}$ when incipient is written $\boldsymbol{Z} . \mathrm{S}$, in the beginning of a word, being never sounded like $Z$, cannot in such case with propriety be substituted for this letter*. In the progress of a word $Z$ is always to be expressed by $S$, which frequently assumes that sound.- Note: the horizontal line of incipient $\mathrm{Z}$ is joined to the succeeding letter.

Here ends the alphabet of consonants; of which it may briefly be said, that of the eighteen general characters exhibited in the "table," I have applied all those that were best calculated for subsequents to letters which indispensably required them, being thirteen in number; viz. BDFGKLM N PR STCh. One more of the eighteen I assigned to Th as an indispensable incipient; and the remaining four, viz. $/ \mathrm{de}-$ scending, $\quad I, P=q, S=\urcorner$ (with the exception of $\uparrow+$ modified) I rejected for the prevention of exceedingly difficult anglesf. What fractional advantage may be gained by an alphabet which shall retain the whole, disposed in regular or-

* The want of this distinction has, more than once, confused me. Context is not at all times immediately decisive.

+ For the sake of lineality I shall employ the rejected loop $P$ similarly modified for the letter $L$ after ascending strokes, as thus $q$ S. However, this modification or'inflection of the line need not be adopted, but when convenience shall require it.

I I have so frequently mentioned angles, that an example may be desired. Take the word "Scotland," as expressed by Dr. Mavor's Alphabet, which no modern contrivance has excelled-and what hand shall execute it with precision and dispatch? The separate letters of which it is composed are Sktlnd $-1 / / \cup)$. 
der of occurrence, without regard to muscular execution or lineality,-let those who may wish to form and professionally to practise such an alphabet, decide.

\section{of Vowers.}

This part of my subject shall be soon discussed. If the immediate reading of any passage promiscuously taken upand not deciphering with a certain share of difficulty, by the aid of context-be the object of the writer; occasional vowels not only in the beginning and ending, but in the middle of words, are indisputably necessary: and to each of these vowels separate characters must assuredly be given. If $\mathrm{Na}$ ture, then, has not furmished us with simple ones, we must either resort to those of a tedious and complex kind ill-calculated for short-hand, or we must avail ourselves of the most eligible consonant characters, distinguishing them as vowels by position*. The sounds of which I have sometimes felt the want, are as follow : $\mathrm{H}$ [or aspirate] $\mathrm{A}, \mathrm{E}, \mathrm{I}=\mathrm{Y}, \mathrm{O}, \mathrm{U}, \mathrm{Oo}$, $\mathrm{Au}, \mathrm{Ou}, \mathrm{Oi}$; also intermediate $\mathrm{Y}$ (as in "beyond"). And if, agreeably to the present plan, intermediate $W$ be added, we shall require no less than eleven characters to represent them. Of these $\mathrm{W}$ and $\mathrm{Y}$ already exist; a simple dot shall stand for $\mathrm{I}=\mathrm{Y}$; complex characters shall for obvious reasons be given to the diphthongs; the aspirate has been already described; and the vowels $A, E, O, U, O o$, must be taken from the class of consonantst.

As the plate with my observations thereon, when presented to the reader, shall exhibit the whole of this scheme, unnecessary anticipation must be here avoided. I shall therefore content myself, on this occasion, with describing the manner of distinguishing those vowels by position; exemplified by the vowel $I=Y$. Let us then so place this vowel with respect to the letters $R T$, that without the possibility of mistake we shall discover the various combinations Irt $\cdot \mathbf{A}$ Rit $\dot{i}$ Rti $\mathbf{A}$. Rtit 1 -

What can be more simple and more perspicuous? In truth I know not any case, intermediate or other, with respect to the placing of these vowels, that presents a difficulty which (unless the consonants be unreasonably small $\ddagger$ or ill-formed)

a mo-

* If, agreeably to modern usage, a dot, by changes of position, shall represent three distinct vowels; why should not a single change of position distinguish a vowel from a consonant?

$+A$ сомMA, and comMa reversed are equivalent to two of these, as shall in due time be explained. Nor shall this comma interfere with our common stop which is called by that name.

$\mp$ As expedition is in some degree promoted by exceedingly small writVol. 61. No. 301. May 1823. 
a moderately intelligent person may not with ease overcome.

I shall now touch on

\section{The Prepositrves.}

These are no more in form than ordinary consonants; but, in consequence of their size and elevation, they become even strikingly conspicuous. They are equivalent to capitals in common writing; and represent, as far as possible, by outline, or rather by some obvious characteristic, the incipient looped characters whose place, for the promotion of brevity, they are intended to supply. The looped incipients are in all but five; of which, Ch occurring too rarely to notice, there are but four to recognise; viz. $G, K, L, P$, which shall be delineated on the plate. $\mathrm{P}$, in the mean time, may serve to elucidate my object, it being written not $P=9$ but $\mid$; as in the words $m$ prt $\cap$ h [ that is, "my part"]. Here the loop appertaining to $\mathrm{P}$ is omitted, and the letter thus represented by its line $e^{*}$ alone. $\mathrm{L}$ is likewise represented by its proper line $/: \mathrm{K}$ by the curve with which it commences 7 ; and $G$, through necessity, not choice, by the reverse of $K$, as $I$.

LXX. On Electricity excited in Paper.

To the Editors of the Philosophical Magazine and Journal.

THE following is an account of an experiment which was suggested by an accidental circumstance. Should you think it worthy of insertion in your valuable publication, it may possibly amuse some of your readers.

If half a sheet of letter paper be made very warm at the fire, then laid flat on the table, and, while held by one of the edges, rubbed hard with a piece of Indian rubber (or elastic gum), it will be observed to stick to the table as if it were wet. When this is observed to be the case, if two opposite edges

ing, it may perhaps be necessary to use this kind of writing while following a rapid speaker. But, on such occasions, we must dispense with almost all the vowels; and even omit so many petty words as well as syllables of our more consequential words ; that deciphering rather than reading is the inevitable result. If, in common long-hand without contractions, our writing (when we are hard pressed) is scarcely legible by ourselves, how must it be with short-hand?

* Incipient $P$ thus written before $S$, which is a hacizontal line, must produce a right angle, in opposition to my general design. It is not, however, incumbent on the practitioner to use either $P$ or any other of the Prepositives : yet I must observe that in my own practice I find them exceedingly convenient; nay, they simplify the writing to my eye. 